wurde. Es erscheint im Zuge der neuen Antiepileptika wichtig, ein Update dieser Studie - auch für die Zukunft - aufzulegen, und die Dinge auch monozentrisch zu betrachten. Im Gegensatz zu Schiller et al. [3, 4] und Luciano et al. [2], die in ihren Arbeiten auch bei späten Behandlungsregimen noch sehr gute Quoten von Anfallsfreiheit erreichten, ist in Kwan und Brodies Arbeit die Chance von Anfallsfreiheit nach dem dritten Regime klein und nach dem vierten Versuch winzig. Andererseits findet er doch $75 \%$ Patienten, die ein gutes oder sehr gutes Outcome haben, so dass sich die ursprünglichen Zahlen von Kwans und Brodies [1] erster Arbeit doch deutlich in Bezug auf das Outcome Anfallsfreiheit gebessert haben. Ein bisschen Zurückhaltung bleibt bei der Einschätzung, wie viel besser die idiopathischen Epilepsien wirklich abschneiden. Es fehlt hier eine Aufstellung darüber, welche idiopathischen Syndrome gemeint sind. Interessant sind auch die Daten zum „sudden unexpected death in epilepsy" (SUDEP). Insgesamt star-

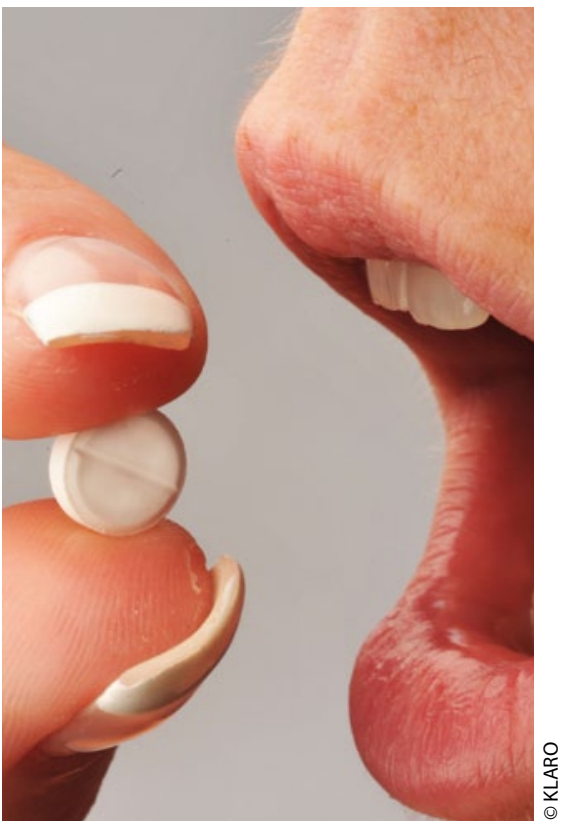

Unter Monotherapie ist die Wahrscheinlichkeit, anfallsfrei zu werden, offenbar höher als unter Kombinationstherapie. ben 76 Patienten in der langen Studienzeit, 46 davon waren anfallsfrei, 30 nicht. Es gab sieben SUDEP, zwei davon betrafen Menschen, die anfallsfrei waren, die anderen Todesfälle wurden als nicht epilepsiebezogen gewertet.

Dr. med. Thomas Mayer

Referenzen:

1. Kwan P, Brodie MJ. Early identification of refractory epilepsy. N Engl J Med. 2000 Feb 3; 342 (5): 314-9

2. Luciano AL, Shorvon SD. Results of treatment changes in patients with apparently drug-resistant chronic epilepsy. Ann Neurol. 2007 Oct; 62(4): 375-81

3. Schiller Y. Seizure relapse and development of drug resistance following long-term seizure remission. Arch Neurol. 2009 Oct; 66 (10): 1233-9

4. Schiller Y, Najjar Y. Quantifying the response to antiepileptic drugs: effect of past treatment history. Neurology. 2008 Jan 1; 70 (1): 54-65

Brodie MJ et al. Patterns of treatment response in newly diagnosed epilepsy. Neurology 2012; 78: 1548-54

\title{
Dem SUDEP auf der Spur
}

Die Suche nach ätiologisch relevanten Faktoren für das Auftreten von plötzlichen unerwarteten Todesfällen bei Epilepsiepatienten (SUDEP = sudden unexpected death in epilepsy) stellt Nervenärzte und Epidemiologen immer noch vor große wissenschaftliche Herausforderungen. Die Autoren der folgenden Studie führten eine Regressionsanalyse einer älteren Studie durch. Diese bestätigt die Annahme aus der Studie von 2011, dass besonders die Anzahl generalisierter tonisch-klonischer Anfälle eng mit der SUDEPRate korreliert ist.

$\mathrm{D}$ ie Autoren, Mitglieder der epidemiologischen Kommission der ILAE (Subcommission on Mortality), hatten 2011 in der zusammenfassenden Analyse von vier Fall-Kontroll-Studien klare Hinweise darauf gefunden, dass vor allem eine hohe Frequenz generalisierter tonisch-klonischer Anfälle und eine antiepileptische Polytherapie mit einer erhöhten SUDEP-Rate assoziiert sind [1]. Als weitere, das Risiko signifikant erhöhende Faktoren, waren unter anderem auch eine lange Epilepsiedauer, männliches Geschlecht und eine symptomatische Ätiologie identifiziert worden [1]. Ungeklärt blieb in dieser vorausgegangenen Arbeit jedoch, wie sich die Faktoren „Frequenz tonisch-klonischer Anfälle“ und „Art und Anzahl der Antiepileptika (AE) " in ihrer Relevanz für das Auftreten von SUDEP unterscheiden.
Basis für die aktuelle multivariate Regressionsanalyse stellten ebenfalls Daten aus vorausgegangenen Fall-KontrollStudien zur Häufigkeit von großen epileptischen Anfällen und zur Behandlung mit Carbamazepin, Phenytoin, Valproat und Lamotrigin beziehungsweise anderen $\mathrm{AE}$ dar. Insgesamt untersuchten die Wissenschaftler 376 Fälle (1.505 Kontrollen). In der nicht-adjustierten Analyse waren folgende Punkte für ein erhöhtes SUDEPRisiko prädisponierend: die Frequenz großer Anfälle, eine Polytherapie und die Anzahl der eingenommenen AE. Nach Adjustierung für den Einflussfaktor „Anzahl von generalisierten Anfällen “ ergab sich hingegen keine Änderung des Risikos durch eine antiepileptische Monotherapie oder Polytherapie beziehungsweise für die individuell untersuchten $\mathrm{AE}$ an einem SUDEP zu versterben.
Kommentar: Zusammenfassend untermauern die Ergebnisse dieser Arbeit die Annahme, dass vor allem die Anzahl von generalisierten tonisch-klonischen Anfällen eng mit der SUDEP-Rate korreliert ist. Nicht bestätigt wurde hingegen die in zahlreichen früheren Arbeiten postulierte Risikoerhöhung durch bestimmte Antiepileptika. Als Fazit fordern die Autoren verstärkte, insbesondere auch pharmakotherapeutische Maßnahmen zur Verringerung von großen epileptischen Anfällen.

Dr. med. Peter Hopp

Referenz:

1. Hesdorffer DC, Tomson T, Benn E, et al. Combined analysis of risk factors for SUDEP. Epilepsia 2011; 52: 1150-59

Hesdorffer DC et al. Do antiepileptic drugs or generalized tonic-clonic seizure frequency increase SUDEP risk? A combined analysis. Epilepsia 2012; 53: 249-52 